\title{
REFLEXÕES TEÓRICAS SOBRE O CUIDAR DE SI À LUZ DO INTERACIONISMO SIMBÓLICO: UM ESTUDO DE REVISÃO INTEGRATIVA
}

\author{
Sheilane da Silva Santos', Glaucia Valente Valadares ${ }^{1}$, George Luiz Alves Santos', Ligia \\ Santana Rosa ${ }^{1}$, Jussara Regina Martins ${ }^{1}$ e Paulo Joaquim Pina Queirós ${ }^{2}$ \\ ${ }^{1}$ Escola de Enfermagem Anna Nery/UFRJ, Brasil. sheilane silva@hotmail.com; gvvufri@gmail.com; \\ georgealvesrad@hotmail.com; lilimatipo@gmail.com; jussaramartinsjf@gmail.com \\ ${ }^{2}$ Escola Superior de Enfermagem de Coimbra, Portugal. pauloqueiros@esenfc.pt
}

\begin{abstract}
Resumo. Introdução: É necessário o investimento no desenvolvimento profissional do enfermeiro, estimulando-o para a prática do cuidar de si, não só proporcionando ao outro um cuidado mais consciente e seguro, mas, também, para proporcionar um nível satisfatório de bem-estar no que tange a sua saúde. Objetivo: discutir os principais achados acerca do cuidado de si em enfermagem à luz do Interacionismo Simbólico. Métodos: revisão integrativa de literatura realizada nas bases de dados LILACS, BDENF, MEDLINE/PubMed e SCIELO, de estudos publicados entre 2014 e 2019, em inglês, português e espanhol. Resultados: foram analisados 12 estudos que abordavam o cuidado de si de forma conceitual, teórica e prática nos diversos cenários que compõem a enfermagem, revelando as relações interpessoais estabelecidas na construção do cuidado fundamental. No que se refere à discussão dos estudos à luz do Interacionismo Simbólico, nenhum artigo utilizou-se deste referencial em suas análises. Conclusão: a presente revisão traz à discussão como o enfermeiro se percebe na perspectiva do cenário de cuidado e como este tem percebido esta relação quando o objeto em questão são suas próprias necessidades de autoconhecimento, revelando aspectos que claramente se relacionam com os conceitos do Interacionismo Simbólico.
\end{abstract}

Palavras-chave: Enfermagem; Equipe de Enfermagem; Relações Interpessoais.

\section{THEORETICAL REFLECTIONS ON TAKING CARE OF ONESELF IN THE LIGHT OF SYMBOLIC INTERACTIONISM}

\begin{abstract}
Introduction: It is necessary to invest in the professional development of nurses, encouraging them to practice taking care of themselves, not only providing others with more conscious and safe care, but also to provide a satisfactory level of well-being in what they do. your health. Objective: to discuss the main findings about nursing self-care in the light of Symbolic Interactionism. Methods: integrative literature review conducted in the LILACS, BDENF, MEDLINE/PubMed and SCIELO databases, of studies published between 2014 and 2019, in English, Portuguese and Spanish. Results: 12 studies were analysed that addressed self-care in a conceptual, theoretical and practical way in the different scenarios that make up nursing, revealing the interpersonal relationships established in the construction of fundamental care. With regard to the discussion of studies in the light of Symbolic Interactionism, no article has used this framework in its analyses. Conclusion: this review brings to discussion how nurses perceive themselves in the perspective of the care scenario and how they perceive this relationship when the object in question is their own needs for self-knowledge, revealing aspects that clearly relate to the concepts of Symbolic Interactionism.
\end{abstract}

Descriptors: Nursing; Nursing, Team; Interpersonal Relations.

\section{INTRODUÇÃO}


O cuidado em enfermagem, segundo Sharovsky et al. (2014, p. 101) representa a "essência da profissão e pertencente a duas esferas distintas: uma objetiva, relacionada ao desenvolvimento de técnicas, bem como procedimentos específicos; e outra esfera, que é a subjetiva, baseada na sensibilidade, criatividade do profissional e das relações intersubjetivas estabelecidas pelo mesmo". Refletindo sobre o cuidado, talvez seja possível compreender que as atitudes dos profissionais de enfermagem precisam ser reavaliadas constantemente, pois, apesar de terem o cuidado como base da profissão, "ainda se deparam com atitudes e práticas que os tornam mecanicistas" (Santos et al., 2017, p.05).

Para tanto, de acordo com Soares, Zeitoune, Lisboa e Mauro (2011, p.42), existe "a real necessidade do investimento no desenvolvimento profissional do enfermeiro", estimulando-o para a prática do cuidar de si, não só proporcionando ao outro um cuidado mais consciente e seguro, mas, também, para proporcionar um nível satisfatório de bem-estar no que tange a sua saúde.

Nessa caminhada podemos observar que a noção de bem-estar, seja ela posta na prática profissional ou no dia a dia pessoal e particular do enfermeiro, reflete o significado que o cuidar de si tem no contexto em que o mesmo se insere. Desta forma, permite-nos discutir sobre este significado com base nos conceitos do referencial teórico Interacionismo Simbólico, que tem sido amplamente utilizado nos estudos de enfermagem. O Interacionismo Simbólico é um referencial que aborda como premissa básica "a construção do significado através das interações sociais, esclarecendo que tais relações são totalmente moldáveis ao longo do tempo na medida em que se estabelecem novos processos interacionais" (Coulon, 1995, p.23).

De acordo com Gabatz, Schwartz e Milbrath (2016, p.369), o "Interacionismo Simbólico foca na natureza da interção social e nela a dinâmica e as atividades sociais tomam lugar entre os atores". Os indivíduos interagem, sendo as sociedades construídas a partir das interações dos indivíduos. A sociedade desta forma sofre constantes mudanças geradas pelos processos interacionais da relação humana.

É importante ressaltar que o cuidado de si vai além de meros procedimentos ou atitudes voltadas para o autocuidado, já que envolve relações que são construídas a partir de dimensões simbólicas que podem ser compreendidas como ações, interações e comportamentos claramente caracterizados pelos preceitos do Interacionismo Simbólico. 
Portanto, é fundamental evidenciar o cuidado de si na perspectiva do seu dinamismo a ser construído através das múltiplas interações num determinado contexto social.

Podemos perceber que existe uma conexão teórica entre o conceito central de significado revelado pelo Interacionismo Simbólico e o conceito de cuidado de si a ser trabalhado em diversas áreas da pesquisa em saúde. Baseado no que aqui foi exposto, temos como objetivo deste artigo: discutir os principais achados acerca do cuidado de si em enfermagem à luz do Interacionismo Simbólico.

\section{MÉTODOS}

Trata-se de um estudo realizado através da revisão integrativa da literatura sobre o cuidado de si na enfermagem. Assim, a revisão integrativa de literatura é um método que tem como finalidade sintetizar resultados obtidos em pesquisas sobre um tema ou questão, de maneira sistemática, ordenada e abrangente. É denominada integrativa porque fornece informações mais amplas sobre um assunto/problema, constituindo, de tal modo, um corpo de conhecimento. Deste modo, no que afirma Ercole, Melo e Alcoforado (2014, p.255), “o revisor/pesquisador pode elaborar uma revisão integrativa com diferentes finalidades, podendo ser direcionada para a definição de conceitos, revisão de teorias ou análise metodológica dos estudos incluídos de um tópico particular".

Para a construção da revisão integrativa é preciso percorrer seis etapas distintas, sendo elas: a identificação do tema e seleção da hipótese ou questão de pesquisa; estabelecimento de critérios para inclusão e exclusão de estudos/amostragem ou busca na literatura; definição das informações a serem extraídas dos estudos selecionados/ categorização dos estudos; avaliação dos estudos incluídos; interpretação dos resultados; e apresentação da revisão/síntese do conhecimento.

Para responder a primeira etapa da revisão integrativa, elaborou-se a questão norteadora como direcionadora do estudo, a saber: como se revelam as produções científicas acerca do cuidado de si em enfermagem?

Em cumprimento a segunda fase da revisão, com finalidade relacionada a amostragem da literatura, foram realizadas buscas nas bases de dados: Literatura Latino-Americana e do Caribe em Ciências da Saúde (LILACS), Bases de Dados de Enfermagem (BDENF), Medical Literature Analysis and Retrieval System Online (MEDLINE/ PubMed) e na biblioteca 
eletrônica Scientific Eletronic Library Online (SciELO) através dos descritores controlados pelos DeCS (Descritores em Ciências da Saúde) "enfermagem", "equipe de enfermagem", "cuidados de enfermagem" e o descritor não controlado "cuidado de si". Estes termos foram utilizados de forma conjunta e isolados.

No que se refere à coleta dos dados correspondente a terceira fase de revisão, as obras pesquisadas foram publicadas no período de 2014 a 2019, sendo eleito este recorte temporal para os estudos com a intencionalidade de abarcar as produções mais recentes acerca do tema. A coleta do material para a pesquisa foi realizada de outubro a dezembro de 2019. Os critérios de inclusão para seleção dos artigos foram: estudos publicados na íntegra entre 2014 e 2019, em português, inglês e espanhol sobre a temática cuidado de si na área de enfermagem.

Excluíram-se os editoriais, resumos de anais, trabalhos de conclusão de curso, boletins epidemiológicos, relatórios de gestão, documentos oficiais de programas nacionais e internacionais, livros, teses e dissertações, publicações que não se enquadraram no recorte temporal estabelecido e estudos que não respondiam ao objetivo proposto inicialmente. Estudos encontrados em duplicidade nas bases de dados foram considerados uma única vez.

Em levantamento bibliográfico preliminar com os descritores elencados, foram reunidos um total de 94 artigos que se assemelhavam a temática descrita para análise. Após leitura de seus títulos e resumos, 36 estudos foram excluídos após terem sido identificadas duplicações dos seus textos, restando um total de 58 artigos.

Nestes foram aplicados os critérios de inclusão e exclusão, onde 27 estudos foram dispensados, restando um total de 31 para leitura de seus textos na íntegra. Então, após leitura dos textos e paralela aplicação da questão norteadora desta pesquisa, tivemos como resultado final 12 estudos para análise e construção desta revisão, conforme ilustra o diagrama abaixo representado pela Figura 1. Este estudo teve sua avaliação crítica realizada por dois de seus autores, de forma independente.

Para a avaliação dos estudos referente à quarta fase do método elaboramos um quadro sinóptico com os principais atributos dos estudos selecionados onde podemos destacar os autores, ano, base indexada e principais benefícios, como demonstrado abaixo no Quadro 1. 
Escolha do tema e

elaboração da questão

norteadora de pesquisa

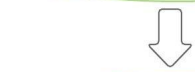

Pesquisas realizadas nas

bases BDENF, SciELO

MEDLINE/Pubmed e LILACS

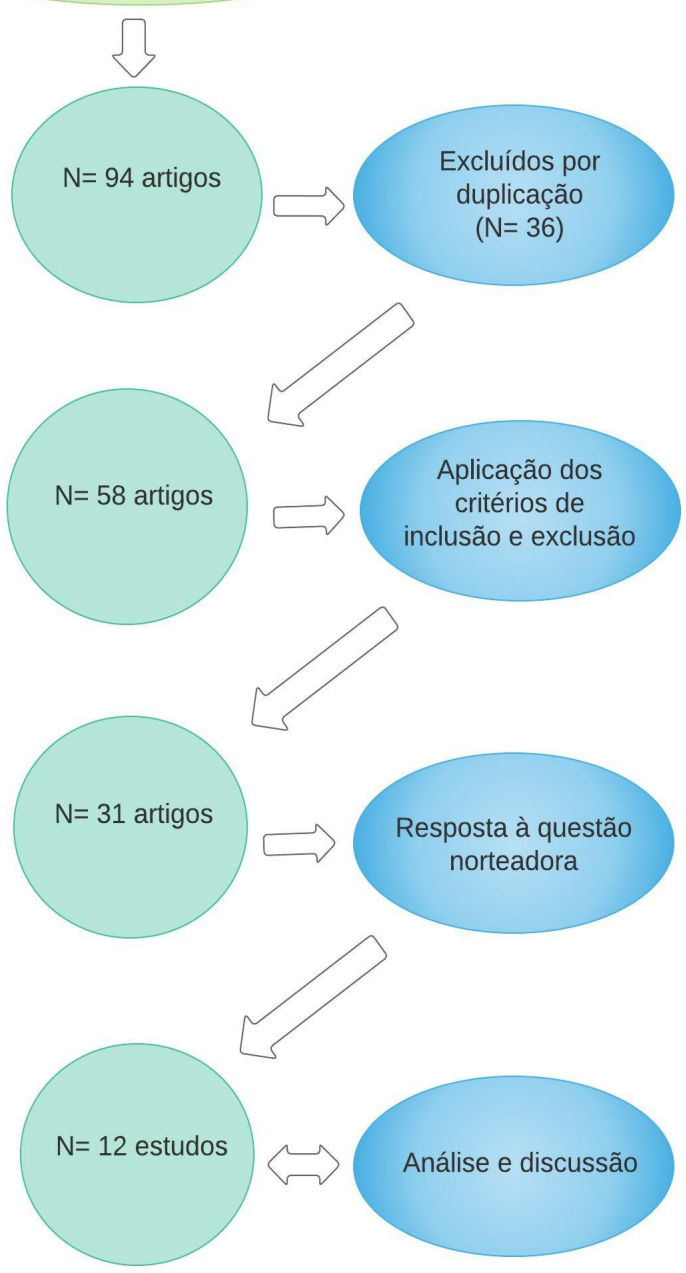

Figura 1. Diagrama das etapas de busca dos estudos. 
Quadro 1. Distribuição das principais características encontradas nos estudos publicados entre 2014 a 2019.

\begin{tabular}{|c|c|c|}
\hline Autores/Ano & $\begin{array}{c}\text { Base } \\
\text { indexada }\end{array}$ & Principais Benefícios \\
\hline $\begin{array}{l}\text { Silva A. A., Terra M. G., Gonçalves M. } \\
\text { O., Souto VT./2014 }\end{array}$ & SciELO & $\begin{array}{c}\text { Demonstra um cuidado prestado ao } \\
\text { outro deslocado de si mesmo. }\end{array}$ \\
\hline $\begin{array}{c}\text { Elias E. A., Souza I. E. O., Vieira } \\
\text { L.B./2014 }\end{array}$ & LILACS & $\begin{array}{l}\text { Expõe o cuidar do outro em detrimento } \\
\text { de si por mulheres. }\end{array}$ \\
\hline Blum C. A./2014 & $\begin{array}{l}\text { MEDLINE/ } \\
\text { PubMed }\end{array}$ & $\begin{array}{l}\text { Reforça a importância do combate ao } \\
\text { estresse no cuidado de si. }\end{array}$ \\
\hline $\begin{array}{l}\text { Oliveira R. K. M., Maia C. A. A. S., } \\
\text { Queiroz J. C./2015 }\end{array}$ & LILACS & $\begin{array}{l}\text { Trata o ambiente de trabalho como } \\
\text { (des)favorecedor do cuidar de si. }\end{array}$ \\
\hline $\begin{array}{l}\text { Ferreira E. S., Souza M. B., Souza N. } \\
\text { V. D. O., Tavares K. F. A., Pires A. } \\
\text { S./2015 }\end{array}$ & BDENF & $\begin{array}{c}\text { Trabalha o cuidado de si sob a ótica do } \\
\text { bem-estar e da busca a qualidade de } \\
\text { vida. }\end{array}$ \\
\hline $\begin{array}{c}\text { Tomasschewski-Barlem J. G., Piexak } \\
\text { D. R. , Barlem E. L. D., Lunardi V. L., } \\
\text { Ramos A. M. /2016 }\end{array}$ & BDENF & $\begin{array}{c}\text { Destaca os aspectos biopsicossocias do } \\
\text { cuidar de si e a importância ao bem- } \\
\text { estar do profissional. }\end{array}$ \\
\hline $\begin{array}{c}\text { Sebold L. F., Kempfer S. S., Girondi J. } \\
\text { B. R., Prado M.L./2016 }\end{array}$ & SciELO & $\begin{array}{l}\text { Destaca o cuidado sob a lógica da } \\
\text { empatia. }\end{array}$ \\
\hline Crane P. J., Ward S.F./2016 & $\begin{array}{l}\text { MEDLINE/ } \\
\text { PubMed }\end{array}$ & $\begin{array}{l}\text { Revela técnicas para o enfermeiro } \\
\text { conhecer-se e cuidar de si. }\end{array}$ \\
\hline $\begin{array}{l}\text { Xavier A.P., Barreto D.M., Alóchio K. } \\
\text { V., Sá S. P. C., Silva Júnior D. I./ } 2017\end{array}$ & BDENF & $\begin{array}{c}\text { Destaque aos principais impeditivos } \\
\text { para o enfermeiro postergar o cuidado } \\
\text { de si. }\end{array}$ \\
\hline $\begin{array}{c}\text { Andrade E.O., Givigi L.R.P., Abrahão } \\
\text { A.L./ } 2018\end{array}$ & LILACS & $\begin{array}{l}\text { Contribui para a discussão das práticas } \\
\text { de produção do cuidado de si pautadas } \\
\text { pelo desafio de se deslocarem da } \\
\text { hegemonia dos referenciais prescritivos } \\
\text { e homogeneizantes no campo da Saúde. }\end{array}$ \\
\hline $\begin{array}{l}\text { Silva M .A., Teixeira E. R., Pereira E. } \\
\text { R., Silva R. M. C. R. A., Rocha R. C. N. } \\
\text { P., Rondon S. O. B./ } 2019\end{array}$ & SciELO & $\begin{array}{l}\text { Analisa a saúde como direito na } \\
\text { perspectiva do cuidado de si, na } \\
\text { concepção dos profissionais de } \\
\text { enfermagem. }\end{array}$ \\
\hline Gomes, I. D./ 2019 & LILACS & $\begin{array}{l}\text { Discuti sobre a enfermagem contar com } \\
\text { o cuidado de si, como um saber que Ihe } \\
\text { é próprio, sendo a aplicação deste saber } \\
\text { em atuação concreta e visível. }\end{array}$ \\
\hline
\end{tabular}

$\mathrm{Na}$ quinta fase da revisão, apresentamos no desenvolvimento do estudo, os principais atributos dos artigos selecionados onde podemos destacar os autores, ano, base indexada e principais benefícios e, posteriormente, a sexta e última fase, onde realizamos a síntese do conhecimento elucidado e sua análise sob a ótica do referencial Interacionismo Simbólico. Isto respondendo ao objetivo proposto inicialmente, além de demonstrar algumas lacunas sobre a produção científica acerca o cuidado e si em enfermagem analisadas com base nos conceitos centrais deste referencial. 


\section{DISCUSSÃO DOS RESULTADOS}

O cuidado, em sua essência na construção conceitual, pode ser percebido como uma forma de agir. Soares e Zeitoune (2012, p.44) afirmam que este é "produzido como experiência do encontro específico e delineado por subjetividades que abarcam diferentes aspectos, a saber: sociais, políticos, culturais, históricos, dentre muitos outros"; que acabam por revelar as interações entre cidadãos em uma determinada sociedade. Um aspecto fundamental do cuidado é tentar compreender a realidade do outro; envolve sair da própria estrutura referencial e buscar a compreensão do outro. Ou seja, é um sentir-se com o outro.

O cuidado que queremos afirmar, de acordo com Andrade, Givigi e Abrahão (2018, p.69):

Aponta para a possibilidade de uma produção singular da/na existência que se abra para diferentes modos de ver, ouvir, pensar, sentir e cuidar. De um cuidado que provoque, nas relações com o outro, possibilidades de deslocamentos, de transformações seja no trabalhador, seja no usuário, de modo que o que vai nortear o cuidado será menos o protocolo, a norma e a regra, e mais a possibilidade de criação que se abre no fazer em saúde a partir de cada encontro.

As ações do cuidar "são reconhecidas socialmente e associadas à natureza do ser humano, portanto, no âmbito sociocultural do exercício do cuidar, percebe-se que o mesmo sempre exigiu o acolhimento de uma individualidade" (Xavier et al., 2017, p.1182). Nesta relação de empatia, o cuidado de enfermagem se revela por agregar fatores que em sua essência vão além das técnicas e práticas "automatizadas". Não pode ser apenas o resultado de um processo de trabalho puramente técnico, bem como nas expertises pessoais segmentadas. Há de tal modo, uma urgente necessidade de revisão que busque fortalecer o cuidado, cada vez mais, na construção do saber profissional, enquanto ciência em contínua evolução.

Como processo de trabalho da enfermagem, o cuidado relacionado às ações de promoção e proteção à saúde deve estar também interligado às questões inerentes ao profissional, a sua percepção sobre o significado do "ser saudável", no âmbito de aspectos que perpassam a manutenção de sua própria saúde, do seu estilo de vida e da compreensão sobre os cuidados voltados a si. Enfim, tem-se um plano filosófico do cuidar como ato essencial a manutenção da vida de todos os partícipes do mesmo que precisa garantir a sua própria existência.

O enfermeiro tem como essência a arte do cuidar, sendo este um cuidado integral e holístico, que se dá através da compreensão da vida humana e do compartilhamento de informações e de sentimentos, que advêm do contato com o cliente no momento em que cuida (sempre único, portanto, cuidado arte). O cuidar, como afirmam Elias, Souza e Vieira (2014, p.416) 
"exige preocupação, conhecimento, dedicação ao próximo e a si mesmo, devendo o cuidador exercitar antes do cuidado do outro o cuidado de si".

O cuidado de si não designa simplesmente uma preocupação, mas todo um conjunto de ocupações ou, também, uma ocupação regrada, um trabalho com procedimentos e objetivos. Sendo assim, Silva et al. (2019, p.163) descreve esse cuidado de si como um "labor que resulta em tempo para praticá-lo em um exercício de si mesmo, e em toda a filosofia antiga, o cuidado de si foi considerado como dever e técnica, contendo um grau de obrigação fundamental".

Considerando tal contexto, o cuidado de si passa a ser entendido "como um processo de subjetivação, no qual estão envolvidos uma variedade de situações e ações que ajudam os trabalhadores no que tange o alcance do bem-estar" (Ferreira et al., 2015, p.979). Numa reflexão mais ampliada, "o exercício de cuidar de si compreende os cuidados apreendidos como imprescindíveis para que os profissionais de enfermagem promovam o equilíbrio social, físico e emocional no desenvolvimento de suas atividades" (Tomasschewski-Barlem, 2016, p. 4630).

Nesse sentido, "o cuidado de si está relacionado com a própria existência, ou o modo de ser dos enfermeiros. Assim, é através deste que os mesmos se revelam nas diferentes realidades de atuação, funções e responsabilidade" (Crane \& Ward, 2016, p. 390). Não obstante, é real e iminente a necessidade dos enfermeiros se reconhecerem como parte do objeto de cuidado, resguardando as necessidades pessoais na construção das práticas voltadas ao seu próprio cuidado, conhecendo seus limites e respeitando-se como ser semelhante ao outro, porém com necessidades e desejos particulares, individuais e distintos.

A prática do cuidar de si deve ser incorporada sistematicamente em todas as áreas de atuação da enfermagem para que este profissional minimize os desgastes físico, emocional e mental. Assim, não podemos entender o cuidado de si como algo a ser prescrito, mas algo a ser conquistado pela buscas do conhecimento interior, pois o cuidado de si "é particularmente uma busca individual, sobretudo que se dá no encontro consigo e com o outro, evidenciando o ser humano como um ser das relações, que se constrói e que se reconstrói" (Oliveira, Maia, \& Queiroz, 2015, p. 2108).

As relações humanas são o ponto chave para a compreensão do cuidado de si. Os profissionais não se cuidam sozinhos, dependem do outro no convívio. O cuidado de si é 
igualmente entendido como possuidor de dimensões pessoais e coletivas, que se expressam na forma como os seres humanos se relacionam consigo mesmos e uns com os outros em suas interações diárias.

Este cuidar de si, segundo Gomes (2019, p. 09):

Não significa que as pessoas fiquem entregues a si próprias; o que pretende e dar ênfase ao processo reflexivo que deve ser promovido na pessoa, para que esta possa ter autonomia e tomar decisões com base em informação esclarecida, qualquer que seja a sua condição de cuidado, no contexto da sua existência.

Num cenário de ampliação e fundamentação da análise das questões inerentes ao cuidado de si e seus componentes, podemos utilizar o referencial denominado Interacionismo Simbólico. O mesmo busca compreender os aspectos principais da conduta humana, a forma como os indivíduos interagem e como essas relações influenciam na formação dos aspectos sociais e na visão de mundo dos sujeitos, agentes sociais continuamente. "Os estudos, por meio desse referencial, devem analisar os processos pelos quais os agentes determinam suas condutas, baseados em suas próprias interpretações do mundo que os rodeiam" (Coulon, 1995, p. 32).

O Interacionismo Simbólico valoriza o sentido que as coisas têm para o comportamento humano. O sentido emerge do processo de interação entre as pessoas e envolve uma interpretação que ocorre em duas etapas. Na primeira instância, de acordo Moura, Kimura e Praça (2010) "o indivíduo mostra para si o sentido das coisas, indicando um processo de interação consigo mesmo. Na segunda instância, o indivíduo seleciona, reavalia e classifica, transformando os sentidos à luz da situação do momento e da direção de sua ação". O significado deste processo de interpretação das relações sociais na construção e valorização do cuidado de si tem ancoragem nas premissas do Interacionismo Simbólico descritas por Blumer em 1937, sociólogo, discípulo de Mead, que deu escopo a este referencial como hoje assim é denominado.

Blumer define as premissas do Interacionismo Simbólico como sendo (Carvalho, Borges, \& Rego, 2010, p.145):

\footnotetext{
O ser humano orienta seus atos em direção às coisas em função do que estas significam para ele, o significado dessas coisas surge como consequência da interação social que cada qual mantém com seu próximo e os significados se manipulam e se modificam mediante um processo interpretativo desenvolvido pela pessoa ao defrontar-se com as coisas que vão encontrando em seu caminho.
} 
A partir desta compreensão, percebe-se que o mundo de significados que cada enfermeiro faz de si como objeto de próprio de cuidado físico, mental e social; está intimamente ligado ao relacionamento com o outro, numa relação existencial complexa. Por fim, através de uma análise interpretativa, o Interacionismo Simbólico cunha o processo de cuidar de si como resultado da rede de significados construídas pelos enfermeiros no cenário social, onde as relações que os mesmos estabelecem com seus pares irão influenciar na sua percepção de mundo, construindo então suas verdades, evidentemente provisórias, com base na significação social, símbolos e significantes.

\section{CONCLUSÃO}

Na síntese dos achados que cunharam a base da construção deste estudo, tem-se destaque as vivências e experiências pessoais dos enfermeiros como condutoras à prática do cuidado de si, quando estas se estabelecem a partir de um processo de autoconhecimento de suas necessidades individuais, de bem-estar e na relação com o outro. Desta forma, possibilita-se a análise destes achados à luz do Interacionismo Simbólico uma vez que a partir de um processo interpretativo, conjugam-se as ações de cuidado numa relação de troca onde os sujeitos passam a interpretar a realidade de si e definir suas prioridades a partir do significado atribuído a realidade social em que se insere.

No que concerne a aplicação deste estudo para o desenvolvimento das investigações qualitativas, observa-se o desafio de caracterizar e aprofundar as discussões sobre o cuidado de si na conjunção dos seus aspectos subjetivos e objetivos na enfermagem, com rigor e método, contudo partindo de uma análise específica deste objeto, no qual se alicerça em ações dialógicas e contextuais.

Torna-se relevante a reflexão analítica do cuidado de si com base nas premissas do Interacionismo Simbólico de forma a preencher as lacunas existentes nas produções científicas no que se refere ao significado de cuidar de si à luz deste referencial de forma a gerar novas discussões e bases para o aprofundamento desta temática, uma vez que não foram encontrados materiais publicados com esta articulação.

\section{REFERÊNCIAS}

Andrade E. O., Givigi L. R. P., \& Abrahão A. L. (2018). The ethics of self-care as a way of creating possibles for work in Health. Interface (Botucatu), 22(64), 67-76.

Carvalho V. D., Borges L. O., \& Rêgo D. P. (2010). Interacionismo simbólico: origens, pressupostos e contribuições aos estudos em Psicologia Social. Revista psicologia, ciência e profissão, 30(1), 146-61. 
Coulon, A. (1995). A Escola de Chicago. Campinas.

Crane P. J., \& Ward S. F. (2016) Self-healing and self-care for nurses. AORN journal, 104(5), 386-400.

Elias E. A., Souza I. E. O, \& Vieira L. B. (2014). Meanings of themselves-care of nursing professional women in a emergency unit. Escola Anna Nery, 18(3), 415-20.

Ercole F. F., Melo L. S, \& Alcoforado C. L. G. C. (2014). Integrative review versus systematic review. Revista Mineira de Enfermagem, 18(1), 251-260.

Ferreira E. S, Souza M. B, Souza N. V .D. O, Tavares K. F. A, \& Pires A. S. (2015). The importance of self care for nursing professionals. Ciência, Cuidado e Saúde, 14(1), 978-85.

Gabatz R. I. B., Schwartz E., \& Milbrath V. M. (2016). O Interacionismo Simbólico no estudo da criança institucionalizada com o seu cuidador. Atas CIAIQ, 2(1), 366-75.

Gomes, I. D. (2019). Promover o cuidado de si: património da enfermagem para o desenvolvimento sustentado, bem-estar e saúde das populações. Pensar Enfermagem, 23(2), 7-15.

Moura E. L., Kimura A. F., \& Praça N. S. (2010). Ser gestante soropositivo para o Vírus da Imunodeficiência Humana: uma leitura à luz do Interacionismo Simbólico. Acta Paulista Enfermagem, 23(2), 206-11.

Oliveira R. K. M., Maia C. A. A. S., Queiroz J. C. (2015). Self-care in nursing: an integrative review. Revista Cuidado é fundamental, 7(1), 2104-12.

Santos A. G., Monteiro C. F. S., Nunes B. M. V. T, Benício C. D. A. V., \& Nogueira L.T. (2017). O cuidado em enfermagem analisado segundo a essência do cuidado de Martin Heidegger. Revista cubana de enfermagem, 33(3), 1-11.

Sebold L. F., Kempfer S. S., Girondi J. B. R., \& Prado M. L. (2016). Perception of nursing faculty on the care: Heidegger constructions. Revista da Escola de Enfermagem da USP, 50(n.esp), 38-45.

Sharovsky L. L., Fortes C., Biliu F., Floriano M., \& Foglino T. (2014). (Des) cuidando de si: como auxiliares de enfermagem percebem a tarefa de cuidar. Estudos Interdisciplinares em Psicologia, 5(2), 96-112.

Silva M. A., Teixeira E. R., Pereira E. R., Silva R. M. C. R. A., Rocha R. C. N.P, \& Rondon S. O. V. (2019). Health as a right and the care of the self: conception of nursing professionals. Revista Brasileira de Enfermagem, $72,159-65$.

Soares R. J. O., \& Zeitoune R. C. G. (2012). O cuidado de si e suas dimensões: subsídios para o cuidar de si de docentes de enfermagem. Revista cuidado é fundamental, 41-44.

Soares R. J. O., Zeitoune R. C. G., Lisboa M. T. L., \& Mauro M. Y. C. (2011). Fatores facilitadores e impeditivos no cuidar de si para docentes de enfermagem. Texto Contexto Enfermagem, 20(4), 758-65.

Tomasschewski-Barlem J. G., Piexak D. R., Barlem E. L. D., Lunardi V. L., \& Ramos A. M. (2016) Scientific production of nursing about self-care: an integrative review. Revista cuidado é fundamental, 8(3), 4629-35.

Xavier A. P., Barreto D. M., Alóchio K. V., Sá S. P. C., \& Silva Júnior D. I. (2017). Ressignificating nursing care: perceptions of a team. Revista de enfermagem da UFPE on line, 11(3),1179-88. 